\title{
A method for the preparation of audio tapes for dichotic listening research
}

\author{
PAUL M. McDONNELL and SANDRA PERUSSE \\ University of New Brunswick, Fredericton, New Brunswick, Canada
}

\begin{abstract}
A simple method for producing audio tapes with temporally synchronized messages is described. The duration and spacing of words can be precisely controlled. This method requires a minimum of elaborate equipment, yet synchronization is very accurate with highly intelligible speech.
\end{abstract}

The construction of tapes for dichotic listening has been a persisting problem. The main objective has been to produce audio tapes with dual messages (two tracks) as nearly synchronized as possible. Messages that are not synchronized may be alternately attended to and thus may not provide an adequate test of the selectivity of attention (Broadbent, 1954).

Solutions to this problem have either been very expensive and complex or have involved simple procedures that sacrifice accuracy. Treisman and Riley (1969) used a computer program to compress or expand recorded digits to $250-\mathrm{msec}$ duration and to synchronize digits. Ling (1971) and others have attempted to synchronize digits by first taping one series and then using the volume-level meter on the recorder to cue the speaker of the second series. Studdert-Kennedy and Shankweiler (1970) modified a two-channel tape deck to permit the length of leader tape passing between two playback heads to be varied until the onsets of two signals recorded on a tape loop coincided. Onset was determined by monitoring the signals on a three-channel event recorder. Nevertheless, signal duration was not precisely controlled.

Complicated techniques involving computer programs have been only moderately satisfactory. Treisman and Riley (1969) apparently distorted the sound quality of the tapes so that phonemical information was lost. Other laboratories have overcome this problem (Yates, 1972). Another problem is that a limited pool of material (about one item/sec) can be used at any one time because of storage limitations and the relatively slow rate of output. Considerable computer central-processing power is needed and not all researchers have access to such facilities.

Simpler procedures have often led to frustration and sizeable amounts of error. These techniques have ranged from the cut-and-splice procedures to recording stimuli on tape in time with a metronome. In our own attempts to use such techniques, errors are rarely less than $50 \mathrm{msec}$.

Rubino (1972) and Yates (1972) provide ingenious ways of constructing dichotic tapes. But neither has produced a long series of dichotic pairs of equal duration both within and between pairs, while at the same time precisely equating the interword interval.

The method described here achieves this degree of uniformity and minimizes experimenter judgment in locating taped signals.

\section{METHOD}

There are basic problems in producing dichotic tapes: equalizing the duration of the two signals and spacing successive pairs of signals. A review of the techniques suggest (e.g., Rubino, 1972) that when synchronization of signal onset has been achieved, signal duration and spacing are not equal. Even when duration is matched within a pair, other pairs differ. The Treisman and Riley (1969) technique is an exception. Rubino's (1972) method requires measuring the beginning and end of a recorded word by marking the tape with a grease pencil. The technique described here enables the user to measure the exact length of recorded tape required.

\section{Construction}

A 36-in. tape loop is constructed using clear leader and a small portion of magnetic tape (1.875 in. long) spliced into the loop. The purpose of the clear leader is to insure that recorded signals do not exceed a specified maximum length. The color contrast of magnetic and clear tape facilitates identification of the beginning and end of the magnetic strip. In this case, the selected speed is $7 \frac{1}{2} \mathrm{in}$./sec. Thus, in order to produce four signals in $1 \mathrm{sec}$, no signal (i.e., portion of magnetic tape) can exceed $1.875 \mathrm{in}$. in length.

One end of the tape loop is fitted to a Sony T366 tape recorder secured to a wooden base. The other end of the loop is fitted around a revolving tape reel mounted on a capstan attached to a block of wood. The separation of recorder and capstan unit can be varied continuously to suit the length of the tape loop.

The tape loop is allowed to revolve with the tape recorder in the record mode. The experimenter then attempts to record a digit (or some other stimulus) as the magnetic portion approaches the record head. A simple way to do this is to repeat continuously the digit as it approaches and crosses the head. The experimenter must attempt to adjust timing and duration of his message so that the entire piece of tape is used. Two criteria are employed to determine if the digit is suitable: The digit is played back to insure that it was completely intelligible and the magnetic section is slowly passed (manually) by the playback head. The volume-level meter has to show pronounced movement over the length of the tape (i.e., deflect up to boundary of black and red zone).

Several attempts may be required to produce a suitable 
digit; in some cases up to $15 \mathrm{~min} /$ digit is required. After a day of practice, digits can generally be recorded in a single try, although some digits are more difficult than others. We use nine monosyllabic digits, 1-10 excluding 7. Certain digits, such as 5, typically take longer than $1 / 4 \mathrm{sec}$ to speak. As a result, initial attempts may produce clipping of the initial morpheme or perhaps the final stop. If this occurs, the recording must be re-done, but the problem is rare. By eliminating clipped stimuli, the recorded digits are as intelligible as any normal recording can be. Since paired items are recorded on separate tracks, pair intelligibility is more affected by the choice of verbal material than by the recording techniques.

Although this method involves trial and error, it insures that the recorded material will be no long than $1 / 4 \mathrm{sec}$ in duration and that the entire magnetic portion will carry a signal. After a set of digits is recorded on precisely measured pieces of magnetic tape, digits are spliced into a continuous series separated with a clear leader tape that can also be precisely measured.

\section{Application}

In our application, digits are presented, typically, at rates of $1,2,3$, and $4 / \mathrm{sec}$. The duration of the signal is always $1 / 4 \mathrm{sec}$, but the length of clear leader separating digits varies. They are $5.625,1.875$, and .625 in. for 1,2 , and 3 digits/sec, respectively. There is no interval for the $4 / \mathrm{sec}$ rate. The small sections of tape are cut to length as accurately as possible, measured with vernier calipers.

A completed sequence contains nine digits and nine intervals. The sequence is measured and then copied onto a single length of tape. An oscilloscope is used to mark the beginning and end of the sequence, although the volume-level meter can be used as well. If the length corresponds to the original, it is spliced out. Several such series of digits are prepared.

The next step is to effect synchronization of the two series. Three tape recorders are used, one of which must have two record heads. A short section of clear leader is taped to the end of each copied sequence; these are joined to make two tape loops each with a recorded series of digits.

Tape loops are played on two recorders and their outputs

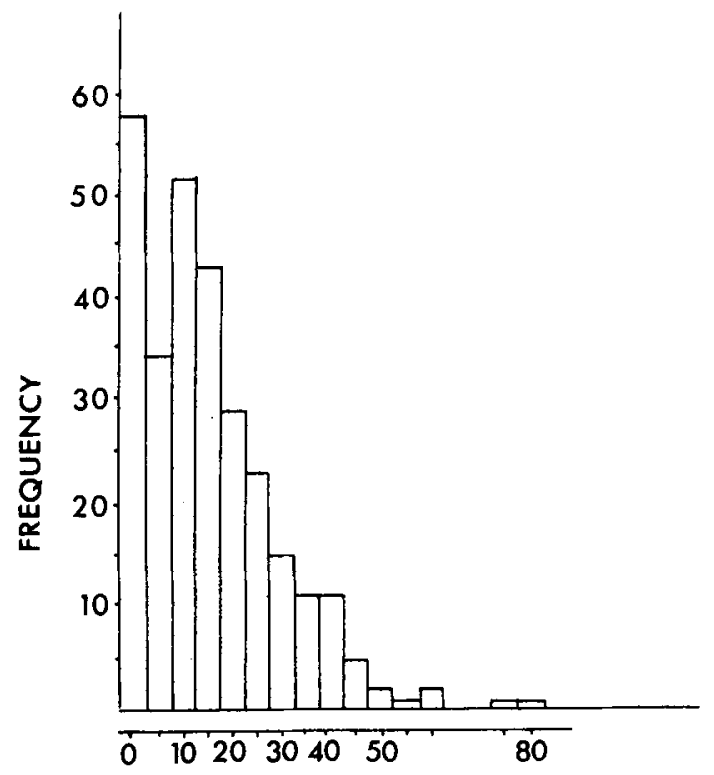

MILLISECONDS

Figure 1. A histogram of synchronization errors measured in milliseconds.
Table 1

Mean, Median, and Mode for Synchronization Errors in Milliseconds

\begin{tabular}{lc}
\hline Measure & Error \\
\hline Mean & 14.6 \\
Median & 10.0 \\
Mode & 0 \\
\hline
\end{tabular}

fed into the third recorder. The three recorders are allowed to operate continuously. By engaging the instant stop on one recorder, the beginning of the two tape loops can be made to coincide. The point of coincidence is monitored by inspecting the level-meter needles of the two-channel recorder. At this point, the experimenter can adjust the record volume of the recording machine to match intensity of the two messages. The original tape loops are reasonably well balanced for intensity by using the automatic record level; also, it is possible to control voice level after some practice (Yates, Smith, Burke, \& Keane, 1969).

Normally this technique of synchronization is inadequate, but since signal duration and spacing are identical for the two tape loops, synchrony can be easily achieved. Of course, evidence is collected to test the amount of error. The new dualmessage recording is subsequently played into two channels of a polygraph or an event recorder set to record at $20 \mathrm{~cm} / \mathrm{sec}$.

\section{RESULTS}

The errors in synchrony are presented in the form of a histogram in Figure 1. The distribution is based on a sample of 108 trials consisting of three samples at each of the four tape speeds, each of which contains nine pairs of digits. Measurements are of both onset and offset errors.

Analysis of variance indicated no significant differences between onset and offset errors $[F(1,182)=.71]$ or between presentation rates $[\mathrm{F}(3,182)=.50]$.

Table 1 gives the mean, median, and mode for the highly skewed distribution in Figure 1. In 20\% of the sample, there was perfect synchrony; in $50 \%$ errors were less than or equal to $1 \mathrm{msec}$; in $80 \%$ errors were less than $25 \mathrm{msec}$; in $95 \%$ errors were less than $50 \mathrm{msec}$.

The degree of accuracy obtained with this technique depends on the user's criterion and intended application. Pairs that demonstrate asynchrony greater than the user's criterion should be eliminated. Practice and experience with the technique results in further error reductions.

\section{REFERENCES}

BroadBent, D. E. The role of auditory localization in attention and memory span. Journal of Experimental Psychology, 1954, 47, 191-196.

Ling, A. H. Dichotic listening in hearing-impaired children. Journal of Speech and Hearing Research, 1971, 14. 793-803.

Rubino, C. A. A simple procedure for constructing dichotic listening tapes. Cortex, 1972, 8, 335-338. 
Studdert-Kennedy, M., \& Shankweiler, D. Hemispheric specialization for speech perception. Journal of the Acoustical Society of America, 1970, 48, 579-594.

Treisman, A., \& Riley, J. Is selective attention selective perception or selective response? A further test. Journal of Experimental Psychology, 1969, 79, 27-34.

Yates, A. J., Smith, P. J., Burke B-D., \& Keane, M. A. A technique for the construction of discrete dichotic stimulation material. Behavior Research Methods \& Instrumentation, 1969, 1, 257-258.

YATES, A. J. Technical, methodological and theorctical problems in dichotic stimulation research. Australian Psychologist, 1972, 7, 2-19.

(Received for publication August 9, 1977; revision accepted October $4,1977$. ) 